BULL. AUSTRAL. MATH. SOC.

VOL. 28 (1983), $411-432$.

\title{
ON APPROXIMATION IN SPACES OF CONTINUOUS FUNCTIONS
}

\author{
HEINZ H, GONSKA
}

\begin{abstract}
This paper deals with approximation of certain operators defined on the space $C(X)$ of real-valued continuous functions on an arbitrary compact metric space $(X, d)$. In particular the problem of giving quantitative Korovkin type theorems for approximation by positive linear operators is solved. This is achieved by using a smoothing approach and the least concave majorant of the modulus of continuity of a function $f$ in $C(X)$. Several new estimates are given as applications, including such for Shepard's method of metric interpolation.
\end{abstract}

\section{Introduction}

The present note deals with quantitative Korovkin type theorems for approximation by positive linear operators defined on $C(X)$. Here $C(X)=C_{\mathbb{R}}((X, d))$ denotes the Banach lattice of real-valued continuous functions defined on the compact metric space $(X, d)$. No further assumptions are made on $X$.

The first such theorem for general positive linear operators and $X=[a, b]$ equipped with the euclidian distance seems to be due to Mamedov [14]. For spaces $(X, d)$ being metrically convex in the sense of Menger [15], Newman and Shapiro [18] proved a theorem similar to that of Mamedov

Received 16 August 1983.

Copyright Clearance Centre, Inc. Serial-fee code: 0004-9727/83 $\$ A 2.00+0.00$. 
(see also Müller and Walk [17, p. 225]). This approach was further developed in two recent papers of Nishishiraho [19, 20] where additional references can be found. For compact spaces $(X, d)$ having a so-called coefficient of convex deformation $\rho<\infty$, Jiménez Pozo [9] published a generalization of the result of Newman and Shapiro mainly involving the modulus of continuity of $f$, given by

$$
\omega(f, \varepsilon):=\sup \{|f(x)-f(y)|: d(x, y) \leq \varepsilon\} .
$$

See $[11,12]$ for a discussion of earlier results.

Furthermore, for arbitrary compact $(X, d)$, Jiménez Pozo [7, 11] proved a generalization of the following

THEOREM A. Let $L: C(X) \rightarrow C(X)$ be a positive linear operator. Then for all $f \in C(X)$, all $x \in X$ and all $\alpha>0$ the following inequality holds:

$$
\begin{aligned}
|L(f, x)-f(x)| \leq w(f, \alpha) \cdot L\left(1_{X}, x\right)+|f(x)| \cdot\left|L\left(1_{X}, x\right)-1\right| & \\
& \quad+\frac{2\|f\|_{X}}{\alpha} \cdot L(d(\cdot, x) ; x) .
\end{aligned}
$$

Here $\|f\|_{X}=\sup \{|f(x)|: x \in X\}$ and $1_{X}: X \ni x \mapsto I \in \mathbb{R}$.

A disadvantage of this type of estimate is the fact that the upper bound is not given in terms of moduli of continuity. The main point of this paper is thus to show that the assumption of metric convexity or of existence of a finite coefficient of convex deformation can be completely dropped when using the least concave majorant of $\omega(f, \cdot)$ instead of $\omega(f, \cdot)$ itself. As will be seen below, our general results imply estimates similar to (and sometimes even better than) those of Nishishiraho and Jiménez Pozo if $(X, d)$ satisfies the assumptions made by them.

It was observed by Berens and Lorentz [1] among others that the results on approximation of lattice homomorphisms $A: C(X) \rightarrow E, E$ a Banach lattice, by positive linear operators are similar to those for approximation of the injection $i: C(X) \rightarrow B(X)$. Here $B(X)$ is the space of bounded real-valued functions on $X$. This is our motivation for proving estimates on approximation of mappings $A: C(X) \rightarrow B(Y), Y \neq \emptyset$, given by $A(f, y)=\Psi_{A}(y) \cdot f\left(g_{A}(y)\right)$, where $\Psi_{A}$ is a bounded real-valued function on $Y$ and $g_{A}$ maps $Y$ into $X$. For the relationship between 
such mappings and lattice homomorphisms see, for example, Wolff [25].

For the sake of brevity we do not strive for utmost generality in this paper. For instance, following the approach of Nishishiraho, more general results can be proved like such involving strictly positive functions $g$ (instead of ${ }^{I_{X}}$ ) and operators $T_{\alpha, \lambda}$. See, for example, [20] for details. It is also possible to drop the assumption of positivity of $L$, or to generalize the operators to be approximated. Some recent results in this direction are due to Jiménez Pozo $[8,10]$ and to the author [3].

\section{Auxiliary results}

As mentioned before our results below will be obtained by using a smoothing approach.

For $0<r \leq 1$ let Lip $r$ denote the set of all functions $g$ in $C(X)$ such that

$$
|g|_{\mathrm{Lip} r}:=\sup _{d(x, y)>0}|g(x)-g(y)| / d^{r}(x, y)<\infty .
$$

Then $\operatorname{Lip} r$ is a dense subspace of $C(X)$, and $|\cdot|_{\text {Lipr }}$ is a seminorm on Lip $r$. Thus it makes sense to use the $K$-functional with respect to (Lip $r,|\cdot|_{\text {Lip } r}$ ) in order to prove quantitative assertions. This functional is given by

$$
K(t, f ; C(X), \operatorname{Lip} r):=\operatorname{in} \hat{r}\left\{\|f-g\|_{X}+t \cdot|g|_{\operatorname{Lip} r}: g \in \operatorname{Lip} r\right\},
$$

where $f \in C(X)$ and $t \geq 0$.

As an immediate consequence of the definition, for any $\lambda \geq 0$ the inequality

$$
K(\lambda t, f ; C(X), \operatorname{Lip} r) \leq \max \{1, \lambda\} \cdot K(t, f ; C(X), \operatorname{Lip} r)
$$

holds.

Another tool for our proofs will be the following lemma of Brudnyí. For a proof see Mitjagin and Semenov [16].

LEMMA 2.1. Every continuous function $f$ on $X$ satisfies

$$
K(\varepsilon / 2, f ; c(X), \operatorname{Lip} l)=\frac{1}{2} \cdot \tilde{\omega}(f, \varepsilon), 0 \leq \varepsilon \leq d(X) .
$$

Here $\tilde{\omega}(f, \cdot)$ denotes the least concave majorant of $\omega(f, \cdot)$ given 
by

$$
\tilde{\omega}(f, \varepsilon)=\left\{\begin{array}{l}
\sup _{0 \leq x \leq \varepsilon \leq y \leq d(X)} \frac{(\varepsilon-x) \omega(f, y)+(y-\varepsilon) \omega(f, x)}{y-x} \text { for } 0 \leq \varepsilon \leq d(X), \\
\tilde{\omega}(f, \varepsilon)=\omega(f, d(X)) \text { if } \varepsilon>d(X),
\end{array}\right.
$$

and $d(X)<\infty$ is the diameter of the compact space $X$.

The definition of $\tilde{\omega}(f, \cdot)$ shows that $\omega(f, \cdot) \leq \tilde{\omega}(f, \cdot)$. However, an estimate from above by some multiple of $\omega(f, \cdot)$ is not possible for all metric spaces $(X, d)$, as will be seen below. For some further properties of $\tilde{\omega}(f, \cdot)$ see, for example, Dzjadyk [2, p. 153ff].

\section{Main theorems}

In this section we prove quantitative theorems on approximation of operators $A$ given by $A(f, y)=\psi_{A}(y) \cdot f\left(g_{A}(y)\right)$ by means of positive linear operators $L$. Here $f \in C(X), Y \neq \emptyset$ is some set, $y \in Y, \psi_{A}$ is a bounded real-valued mapping on $Y$ and $g_{A}$ maps $Y$ into $X$. The key result of this section is

THEOREM 3.1. Let $A$ be given as above and $L \neq 0$ be a positive linear operator both mapping $C(X)$ into $B(Y)$. Then the following inequality holds for all $f \in C(X), y \in Y$, and $\varepsilon>0$ :

$$
\begin{aligned}
|L(f, y)-A(f, y)| \leq \max \left[\|L\|, \frac{1}{\varepsilon} L\left(d\left(\cdot, g_{A}(y)\right) ; y\right)\right] \cdot \tilde{\omega}(f, \varepsilon) & \\
& +\left|L\left(1_{X}, y\right)-A\left(I_{X}, y\right)\right| \cdot\left|f\left(g_{A}(y)\right)\right|
\end{aligned}
$$

Proof. Let $A$ be given by $A(f, y)=\psi_{A}(y) \cdot f\left(g_{A}(y)\right)$, and let $L: C(X) \rightarrow B(Y)$ be positive and linear. Then we have

$$
A(f, y)=\psi_{A}(y) \cdot f\left(g_{A}(y)\right)=A\left(I_{X}, y\right) \cdot f\left(g_{A}(y)\right) .
$$

Thus, for all $f \in C(X)$ and all $y \in Y$,

$$
\begin{aligned}
|L(f, y)-A(f, y)| \leq\left|L(f, y)-L\left(I_{X}, y\right) \cdot f\left(g_{A}(y)\right)\right| & \\
& +\left|L\left(1_{X}, y\right)-A\left(I_{X}, y\right)\right| \cdot\left|f\left(g_{A}(y)\right)\right| .
\end{aligned}
$$

The second term on the right hand side is all right. 
Defining $\tilde{A}(f, y):=L\left(I_{X}, y\right) \cdot f\left(g_{A}(y)\right)$, we have to consider $|L(f, y)-\tilde{A}(f, y)|$. Note that $L\left(1_{X}\right)=\tilde{A}\left(1_{X}\right)$.

Let $g$ be arbitrarily given in Lip 1 . Then

$$
|L(f, y)-\tilde{A}(f, y)| \leq|(L-\tilde{A})(f-g, y)|+|(L-\tilde{A})(g, y)| .
$$

For the first term on the right hand side obviously

$$
\begin{aligned}
|(L-\tilde{A})(f-g, y)| & \leq|L(f-g, y)|+|\tilde{A}(f-g, y)| \\
& \leq 2 \cdot\|L\| \cdot\|f-g\|_{X} .
\end{aligned}
$$

For the second term one gets

$$
\begin{aligned}
|(L-\tilde{A})(g, y)| & =\left|L(g, y)-L\left(I_{X}, y\right) \cdot g\left(g_{A}(y)\right)\right| \\
& =\left|L\left(g-g\left(g_{A}(y)\right\}, y\right\}\right| \\
& \leq L\left(\left|g-g\left(g_{A}(y)\right)\right|, y\right) \\
& \leq|g|_{L i p l} \cdot L\left(d\left(\cdot, g_{A}(y)\right) ; y\right) .
\end{aligned}
$$

Thus we have for any $g$ in Lip I the inequality $|L(f, y)-\tilde{A}(f, y)|$

$$
\begin{aligned}
& \leq 2 \cdot\|L\| \cdot\|f-g\|_{X}+L\left(d\left(\cdot, g_{A}(y)\right) ; y\right) \cdot|g|_{\text {Lipl }} \\
& =2 \cdot\|L\| \cdot\left\{\|f-g\|_{X}+\frac{1}{2 \cdot\left\|_{L}\right\|} \cdot L\left(d\left(\cdot, g_{A}(y)\right) ; y\right\} \cdot|g|_{\text {Lipl }}\right\} .
\end{aligned}
$$

This implies

$$
\begin{aligned}
|L(f, y)-\tilde{A}(f, y)| & \\
& \leq 2 \cdot\|L\| \cdot k\left(\frac{1}{2 \cdot\|L\|} \cdot L\left(d\left(\cdot, g_{A}(y)\right) ; y\right), f ; C(X), \operatorname{Lip} 1\right) .
\end{aligned}
$$

Now introducing an arbitrary number $0<\varepsilon \leq d(X)$ we obtain

$$
\begin{aligned}
\mid L(f, & y)-\tilde{A}(f, y) \mid \\
& \leq 2 \cdot\|L\| \cdot K\left(\frac{1}{\|L\|} \cdot L\left(d\left(\cdot, g_{A}(y)\right) ; y\right) \cdot \frac{1}{\varepsilon} \cdot \frac{\varepsilon}{2}, f ; C(X), \text { Lip } 1\right) \\
& \leq 2 \cdot\|L\| \cdot \max \left\{1, \frac{1}{\|L\| \cdot \varepsilon} \cdot L\left(d\left(\cdot, g_{A}(y)\right) ; y\right)\right\} \cdot K\left(\frac{\varepsilon}{2}, f ; C(X), \text { Lip } 1\right) .
\end{aligned}
$$

Brudnyì's Lemma 2.1 shows that $K(\varepsilon / 2, f ; C(X), \operatorname{Lip} 1)=\frac{1}{2} \cdot \tilde{\omega}(f, \varepsilon)$ which yields

$$
|L(f, y)-\tilde{A}(f, y)| \leq \max \left\{\|L\|, \frac{1}{\varepsilon} \cdot L\left(d\left(\cdot, g_{A}(y)\right\} ; y\right)\right\} \cdot \tilde{\omega}(f, \varepsilon) .
$$


If $\varepsilon>d(X)$ the estimate is also correct since in this case

$$
\begin{aligned}
& |L(f, y)-\tilde{A}(f, y)| \\
& \leq 2 \cdot\|L\| \cdot K\left(\frac{1}{2 \cdot\|L\|} \cdot L\left(d\left(\cdot, g_{A}(y)\right) ; y\right) \cdot \frac{2}{d(X)} \cdot \frac{d(X)}{2}, f ; C(X), \text { Lip } 1\right) \\
& \leq 2 \cdot\|L\| \cdot \max \left\{1, \frac{1}{\|L\|} \cdot L\left(d\left(\cdot, g_{A}(y)\right) ; y\right) \cdot \frac{1}{d(X)}\right\} \\
& \quad \cdot K\left(\frac{d(X)}{2}, f ; C(X), \text { Lip } 1\right\} \\
& =\max \left\{\|L\|, \frac{1}{d(X)} \cdot L\left(d\left(\cdot, g_{A}(y)\right) ; y\right)\right\} \cdot \tilde{\omega}(f, d(X)) \\
& \quad=\max \left\{\|L\|, \frac{1}{\varepsilon} \cdot L\left(d\left(\cdot, g_{A}(y)\right) ; y\right)\right\} \cdot \tilde{\omega}(f, \varepsilon) .
\end{aligned}
$$

Here the last equation follows from the fact that for $\varepsilon>d(X)$ we have $1 / \varepsilon \cdot L\left(d\left(\cdot, g_{A}(y)\right) ; y\right) \leq\|L\|$ and $\tilde{\omega}(f, \varepsilon)=\tilde{\omega}(f, d(x))$. Both observations imply the inequality in Theorem 3.1 .

The following corollary gives an analogue of Theorem 3.1 for a.wider class of test functions.

COROLLARY 3.2. Let the assumptions of Theorem 3.1 be fulfizled. Let $\Phi$ be a fronction on $x^{2}$ such that $\Phi(\cdot, y) \in C(X)$ for all $y \in X$. Moreover, we assume that for some $q \geq 1$ the condition

$$
d(x, y)^{q} \leq \Phi(x, y) \text { for all } x, y \in X
$$

is satisfied. Then

$$
\begin{aligned}
|L(f, y)-A(f, y)| \leq \max \left[\|L\|, \frac{I}{\varepsilon} \cdot C(L, \Phi, A, y)\right] \cdot \tilde{\omega}(f, \varepsilon) & \\
& +\left|L\left(1_{X}, y\right)-A\left(1_{X}, y\right)\right| \cdot\left|f\left(g_{A}(y)\right)\right|,
\end{aligned}
$$

where

$$
C(L, \Phi, A, y):=\inf _{p \geq 1}\left\{L\left(\Phi\left(\cdot, g_{A}(y)\right)^{p / q} ; y\right)^{1 / p} \cdot L\left(1_{X} ; y\right)^{1-1 / p}\right\}
$$

Proof. It is only necessary to estimate $L\left(d\left(\cdot, g_{A}(y)\right) ; y\right)$ in order to obtain the claim of Corollary 3.2 .

For $y$ fixed, the functional $L(*, y)$ is a positive linear form on $C(X)$. Thus if $q \geq 1$, Hölder's inequality implies 


$$
L\left(d\left(\cdot, g_{A}(y)\right) ; y\right) \leq L\left(d\left(\cdot, g_{A}(y)\right)^{p} ; y\right)^{1 / p} \cdot L\left(1_{X} ; y\right)^{1-1 / p} .
$$

If $q \geq 1$ is fixed and such that

$$
d^{q}(x, y) \leq \Phi(x, y) \text { for all }(x, y) \in x^{2},
$$

we have

$$
\begin{aligned}
L\left(d\left(\cdot, g_{A}(y)\right) ; y\right) & \leq L\left(d\left(\cdot, g_{A}(y)\right)^{p} ; y\right)^{1 / p} \cdot L\left(1_{X} ; y\right)^{1-1 / p} \\
& \leq L\left(\Phi\left(\cdot, g_{A}(y)\right)^{p / q} ; y\right)^{1 / p} \cdot L\left(1_{X} ; y\right)^{1-1 / p} .
\end{aligned}
$$

Hence

$$
L\left(d\left(\cdot, g_{A}(y)\right) ; y\right) \leq \inf _{p \geq 1}\left\{L\left(\Phi\left(\cdot, g_{A}(y)\right)^{p / q} ; y\right)^{I / p} \cdot L\left(1_{X} ; y\right)^{1-1 / p}\right\}
$$

Combining the above inequalities we arrive at

$$
\begin{aligned}
& |L(f, y)-A(f, y)| \\
& \leq \max \left[\|L\|, \frac{1}{\varepsilon} \cdot \inf _{p \geq 1}\left\{L\left(\Phi\left(\cdot, g_{A}(y)\right)^{p / q} ; y\right)^{I / p} \cdot L\left(I_{X} ; y\right)^{1-1 / p}\right\}\right] \cdot \tilde{\omega}(f, \varepsilon) \\
& +\left|L\left(I_{X}, y\right)-A\left(I_{X}, y\right)\right| \cdot\left|f\left(g_{A}(y)\right)\right|,
\end{aligned}
$$

which is the claim of the corollary.

For the special case $Y=X, A(f, x)=f(x), L I_{X}=I_{X}$ the inequality in Corollary 3.2 reduces to the one in

COROLLARY 3.3. Under the assumptions just mentioned one has

$$
|L(f, x)-f(x)| \leq \max \left[1, \frac{l}{\varepsilon} \cdot \inf _{p \geq 1}\left\{L\left(\Phi(\cdot, x)^{p / q} ; x\right)^{1 / p}\right\}\right] \cdot \tilde{\omega}(f, \varepsilon) .
$$

Our next theorem is a generalization of Theorem 3.1. It shows that this theorem implies a variety of estimates including uniform ones or such in $L_{1}$ spaces.

THEOREM 3.4. Let $(X, d)$ be a compact metric space and $Y \neq \emptyset$ be some set. Let $L \neq 0$ be a positive linear operator and $A$ be given by $A f=\psi_{A} \cdot\left(f \circ g_{A}\right)$, both mapping $C(X)$ into $B(Y)$. Moreover, let $M=\{\mu\}$ be a set of positive linear functionals on $B(Y)$ such that 
$p_{M}[h]:=\sup \{\mu(|h|): \mu \in M\}<\infty$ for all $h \in B(Y)$, and $\left.p_{M}[]_{Y}\right]>0$.

Then for all $f \in C(X)$ and all $\varepsilon>0$ the following inequality holds:

$$
\begin{aligned}
p_{M}[L f-A f] \leq p_{M}\left[\left(L 1_{X}-A 1_{X}\right) \cdot\left(f \circ g_{A}\right)\right] & \\
& +\max \left\{\|L\| \cdot p_{M}\left[1_{Y}\right], \frac{1}{\varepsilon} \cdot p_{M}\left[L\left(d\left(\cdot, g_{A}(*)\right) ; *\right)\right]\right\} \cdot \tilde{\omega}(f, \varepsilon) .
\end{aligned}
$$

Here $L$ is applied with respect to the variable indicated by "." , and $\mu \in M$ is applied with respect to "*".

Proof. If $g \in$ Lip 1 is arbitrarily given, then the proof of Theorem 3.1 shows that for all $y \in Y$ we have

$$
\begin{aligned}
|(L-A)(f, y)| \leq\left|(L-A)\left(I_{X}, y\right)\right| \cdot\left|f\left(g_{A}(y)\right)\right| \\
+2 \cdot\|L\| \cdot\|f-g\|_{X}+L\left(d\left(\cdot, g_{A}(y)\right) ; y\right) \cdot|g|_{\text {Lipl }} .
\end{aligned}
$$

Applying $\mu \in M$ to this inequality means

$$
\begin{aligned}
& \mu(|L f-A f|) \\
& \leq \mu\left(\left|L 1_{X}-A 1_{X}\right| \cdot\left|f \circ g_{A}\right|\right)+2 \cdot\|L\| \cdot\|f-g\|_{X} \cdot \mu\left(1_{Y}\right)
\end{aligned}
$$$$
+\mu\left(L\left(d\left(\cdot, g_{A}(*)\right) ; *\right)\right) \cdot|g|_{\text {Lipl }}
$$

$\leq p_{M}\left[\left(L 1_{X}-A 1_{X}\right) \cdot\left(f \circ g_{A}\right)\right]$

$+2 \cdot\|L\| \cdot p_{M}\left[I_{Y}\right]\left\{\|f-g\|_{X}+\frac{1}{2 \cdot\|L\| \cdot p_{M}\left[I_{Y}\right]} \cdot p_{M}\left[L\left(d\left(\cdot, g_{A}(*)\right) ; *\right)\right] \cdot|g|_{\text {Lipl }}\right\}$.

Thus

$$
\begin{aligned}
& \mu(|L f-A f|) \leq p_{M}\left[\left(L I_{X}-A 1_{X}\right) \cdot\left(f \circ g_{A}\right)\right] \\
& +2 \cdot\|L\| \cdot p_{M}\left[I_{Y}\right] K\left(\frac{1}{2 \cdot\|L\| \cdot p_{M}\left[I_{Y}\right]} \cdot p_{M}\left[L\left(d\left(\cdot, g_{A}(*)\right) ; *\right)\right], f ; C(X), \text { Lip } 1\right) .
\end{aligned}
$$

Proceeding exactly as in the proof of Theorem 3.1 yields

$$
\begin{aligned}
&\left.\mu(|L f-A f|) \leq p_{M}\left[(L]_{X}-A 1_{X}\right) \cdot\left(f \circ g_{A}\right)\right] \\
&\left.+\max \left\{\|L\| \cdot p_{M}[]_{Y}\right], \frac{1}{\varepsilon} \cdot p_{M}\left[L\left(d\left(\cdot, g_{A}(*)\right) ; *\right)\right]\right\} \cdot \tilde{\omega}(f, \varepsilon) .
\end{aligned}
$$

Passing to the supremum in $M$ now gives the estimate in Theorem 3.4 .

COROLLARY 3.5. If the assumptions of Theorem 3.4 are fulfilled, and if $\Phi$ is given as in Corolzary 3.2, then 


$$
\begin{aligned}
p_{M}[L f-A f] \leq p_{M}\left[\left(L 1_{X}-A \perp_{X}\right) \cdot(\right. & \left.\left.f \circ g_{A}\right)\right] \\
& +\max \left\{\|L\| \cdot p_{M}\left[\left[_{Y}\right], \frac{1}{\varepsilon} \cdot C(M, L, \Phi, A)\right\} \cdot \tilde{\omega}(f, \varepsilon),\right.
\end{aligned}
$$

where

$$
C(M, L, \Phi, A)=\inf _{p \geq 1}\left\{\left(p_{M}\left[L\left(\Phi\left(\cdot, g_{A}(*)\right)^{p / q} ; *\right]\right]\right)^{1 / p} \cdot\left(p_{M}\left[L\left(I_{X} ; *\right]\right]\right)^{1-1 / p}\right\} .
$$

Proof. The assertion in Corollary 3.5 results from an estimate of $p_{M}\left[L\left(d\left(\cdot, g_{A}(*)\right) ; *\right)\right]$ in Theorem 3.4 . For any $p \geq 1$ we have

$$
p_{M}\left[L\left(d\left(\cdot, g_{A}(*)\right) ; *\right)\right] \leq p_{M}\left[L\left(\Phi\left(\cdot, g_{A}(*)\right)^{p / q} ; *\right)^{I / p} \cdot L\left(I_{X} ; *\right)^{1-1 / p}\right] .
$$

Applying Hölder's inequality again, we conclude as in the proof of Theorem 3.4 that

$$
\begin{aligned}
p_{M}\left[L \left(\Phi\left(\cdot, g_{A}(*)\right)^{p / q} ;\right.\right. & \left.*)^{1 / p} \cdot L\left(I_{X} ; *\right)^{1-1 / p}\right] \\
& \leq\left(p_{M}\left[L\left(\Phi\left(\cdot, g_{A}(*)\right)^{p / q} ; *\right]\right]\right)^{1 / p} \cdot\left(p_{M}\left[L\left(I_{X} ; *\right)\right]\right)^{1-1 / p} .
\end{aligned}
$$

Passing to the inf over all $p \geq 1$ implies

$p_{M}\left[L\left(d\left(\cdot, g_{A}(*)\right) ; *\right)\right]$

$$
\leq \inf _{p \geq 1}\left\{\left(p_{M}\left[L\left(\Phi\left(\cdot, g_{A}(*)\right)^{p / q} ; *\right)\right]\right]^{1 / p} \cdot\left(p_{M}\left[L\left(I_{X} ; *\right)\right]\right)^{1-1 / p}\right\} .
$$

This yields the claim of Corollary 3.5 .

If in Theorem 3.4 or Corollary $3.5, M=\left\{\varepsilon_{y}\right\}$ for some point evaluation functional $\varepsilon_{y}, y \in Y$, then the estimates given reduce to the ones in Theorem 3.1 and Corollary 3.2 , respectively.

REMARK 3.6. The estimates given in Theorems 3.1 and 3.4 are best possible in a certain sense. To show this, let $Y=\left\{x_{0}\right\}$, $A\left(f, x_{0}\right)=f\left(x_{0}\right)$, and $L\left(1, x_{0}\right)=1$ for some fixed point $x_{0}$ in $X$. In this case the inequality in Theorem 3.1 (or the one in Theorem 3.4 for $M=\left\{\varepsilon_{x_{0}}\right\}$ reduces to 


$$
\left|L\left(f, x_{0}\right)-f\left(x_{0}\right)\right| \leq \max \left\{1, \frac{1}{\varepsilon} \cdot L\left(d\left(\cdot, x_{0}\right) ; x_{0}\right)\right\} \cdot \tilde{\omega}(f, \varepsilon) .
$$

For $L\left(d\left(\cdot, x_{0}\right) ; x_{0}\right)=0$ we have $\left|L\left(f, x_{0}\right)-f\left(x_{0}\right)\right| \leq \tilde{\omega}(f, \varepsilon)$ for all $\varepsilon>0$ and thus

$$
\left|L\left(f, x_{0}\right)-f\left(x_{0}\right)\right| \leq \tilde{\omega}\left(f, L\left(d\left(\cdot, x_{0}\right) ; x_{0}\right)\right) .
$$

If $L\left(d\left(\cdot, x_{0}\right) ; x_{0}\right)>0$, then choose $\varepsilon=L\left(d\left(\cdot, x_{0}\right) ; x_{0}\right)$, and this gives the same inequality.

Now take $f(\cdot)=d\left(\cdot, x_{0}\right)$. Hence

(3.1) $\left|L\left(d\left(\cdot, x_{0}\right) ; x_{0}\right)-d\left(x_{0}, x_{0}\right)\right|$

$$
=L\left(d\left(\cdot, x_{0}\right) ; x_{0}\right) \leq \tilde{\omega}\left(d\left(\cdot, x_{0}\right), L\left(d\left(\cdot, x_{0}\right) ; x_{0}\right)\right) .
$$

If for instance $X=[a, b]$ and $d(x, y)=|y-x|$, then

$$
\omega\left(\left|\cdot-x_{0}\right|, h\right)=\min \left\{h, \max \left\{b-x_{0}, x_{0}-a\right\}\right\}
$$

for each $h \in[0, b-a]$. Thus $\omega\left(\left|\cdot-x_{0}\right|, h\right)$ is a concave function and

$$
\tilde{\omega}\left(\left|\cdot-x_{0}\right|, h\right)=\omega\left(\left|\cdot-x_{0}\right|, h\right) \text {. }
$$

Hence inequality (3.1) becomes an equality, which shows that the constant 1 in

$$
\left|L\left(f, x_{0}\right)-f\left(x_{0}\right)\right| \leq 1 \cdot \tilde{\omega}\left(f, L\left(d\left(\cdot, x_{0}\right) ; x_{0}\right)\right\}
$$

cannot be improved in general.

We shall now look at spaces $(X, d)$ with the following property: there exists a constant $\eta>0$ such that for all $\xi, \varepsilon>0$ and all $f \in C(X)$, the inequality

$$
\omega(f, \xi \cdot \varepsilon) \leq(1+n \xi) \cdot \omega(f, \varepsilon)
$$

holds.

Examples of such spaces are, for example, compact metric spaces being convex in the sense of Menger [15], or compact convex subsets $(X, d)$ of a metric linear space $(Y, d)$ with translation invariant metric and starshaped $d(\cdot, 0)$ (see Nishishiraho [20]). In both cases the above inequality holds for $\eta=1$. Other examples are given by spaces $(X, d)$ 
having a coefficient of convex deformation $\rho=\rho(X)$. In this case the above inequality holds for $\eta=\rho$ (see Jiménez Pozo [9, Théorème 2]).

In these cases $\tilde{\omega}(f, \cdot)$ and $\omega(f, \cdot)$ are related in the following way .

LEMMA 3.7. If $(X, d)$ is a compact metric space such that (3.2) holds, then for $f \in C(X)$ there holds

$$
\omega(f, \varepsilon) \leq \tilde{\omega}(f, \varepsilon) \leq(I+\eta) \cdot \omega(f, \varepsilon), \varepsilon \geq 0 .
$$

Proof. As mentioned earlier,

$$
\tilde{\omega}(f, \varepsilon)=\sup _{0 \leq x \leq \varepsilon \leq y \leq d(X)} \frac{(\varepsilon-x) \omega(f, y)+(y-\varepsilon) \omega(f, x)}{y-x} .
$$

Putting, for example, $x=\varepsilon$ shows that $\omega(f, \varepsilon) \leq \tilde{\omega}(f, \varepsilon)$.

For the proof of the second inequality let $0 \leq x<\varepsilon<y$. Write $\omega(\varepsilon)=\omega(f, \varepsilon)$ for the sake of brevity. Then

$$
\begin{aligned}
\frac{\varepsilon-x}{y-x} \cdot \omega(y)+\frac{y-\varepsilon}{y-x} \cdot \omega(x) & \leq \frac{\varepsilon-x}{y-x}(n \cdot y+\varepsilon) \varepsilon^{-1} \cdot \omega(\varepsilon)+\frac{y-\varepsilon}{y-x} \cdot \omega(\varepsilon) \\
& \leq \frac{\omega(\varepsilon)}{y-x}(y-x+n(y-x)) \\
& =(1+n) \cdot \omega(\varepsilon) .
\end{aligned}
$$

This yields the estimate from above for $0 \leq \varepsilon \leq d(X)$. Note that it is also true for $\varepsilon>d(X)$.

REMARK 3.8. The second inequality in Lemma 3.7 does not hold for an arbitrary compact metric space. This can be seen from the following simple example.

Let $X=\left[0, \frac{1}{4}\right] \cup\left[\frac{3}{4}, 1\right]$ and $d(x, y)=|x-y|$ for $x, y \in X$. The function $f$ given by

$$
f(x)=\left\{\begin{array}{lll}
1 & \text { for } 0 \leq x \leq \frac{1}{4}, \\
2 & \text { for } \frac{3}{4} \leq x \leq 1,
\end{array}\right.
$$

is continuous on $X$. Its modulus of continuity is

$$
\omega(f, \varepsilon)= \begin{cases}0 & \text { for } 0 \leq \varepsilon<\frac{1}{2}, \\ 1 & \text { for } \frac{1}{2} \leq \varepsilon \leq 1 .\end{cases}
$$


Thus the least concave majorant $\tilde{\omega}(f, \cdot)$ is the function $\tilde{\omega}(f, \varepsilon)=\min \{2 \varepsilon, 1\}$. Hence $\tilde{\omega}(f, \varepsilon) \leq c \cdot \omega(f, \varepsilon)$ cannot hold for any $c>0$ and all $\varepsilon \geq 0$. In fact, it is easily verified that there is no $\eta>0$ such that for all $\xi, \varepsilon>0$ we have

$$
\omega(f, \xi \cdot \varepsilon) \leq(1+n \xi) \cdot \omega(f, \varepsilon) .
$$

Using Lemma 3.7 it is now easy to give estimates involving $\omega(f, \varepsilon)$ itself. We restrict ourselves to quote the following consequence of Theorem 3.4 .

THEOREM 3.9. Let $(X, d)$ be a compact metric space satisfying (3.2) and let the assumptions of Theorem 3.4 be satisfied.

If the fronction $f \in C(X)$ has a concave modulus of continuity, then

$$
\begin{aligned}
& p_{M}[L f-A f] \leq p_{M}\left[\left(L 1_{X}-A 1_{X}\right) \cdot\left(f \circ g_{A}\right)\right] \\
& \quad+\max \left\{\|L\| \cdot p_{M}\left[1_{Y}\right], \frac{1}{\varepsilon} \cdot p_{M}\left[L\left(d\left(\cdot, g_{A}(*)\right) ; *\right)\right]\right\} \cdot \omega(f, \varepsilon) .
\end{aligned}
$$

Otherwise we have

$$
\begin{aligned}
p_{M}[L f-A f] \leq & p_{M}\left[\left(L 1_{X}-A 1_{X}\right) \cdot\left(f \circ g_{A}\right)\right] \\
& +(1+n) \cdot \max \left\{\|L\| \cdot p_{M}\left[1_{Y}\right], \frac{1}{\varepsilon} \cdot p_{M}\left[L\left(d\left(\cdot, g_{A}(*)\right) ; *\right)\right]\right\} \cdot \omega(f, \varepsilon) .
\end{aligned}
$$

REMARK 3.10. (i) In order to compare the result in Theorem 3.9 to one obtained by Nishishiraho [19], we consider the following situation: $Y=X, M=\left\{\varepsilon_{x}\right\}$ for some fixed point evaluation functional $\varepsilon_{x}$, $A(f, x)=f(x), \quad L 1_{X}=1_{X}$. Then for an $f \in C(X)$ having a concave modulus of continuity the above inequality yields, for any $\varepsilon>0$,

$$
|L(f, x)-f(x)| \leq \max \left\{1, \frac{1}{\varepsilon} \cdot L(d(\cdot, x) ; x)\right\} \cdot \omega(f, \varepsilon) .
$$

If in Lemma 4 of $[19]$ we take $g=1_{X}$ and $\Phi(x, y)=d(x, y)$, then the lemma implies

$$
|L(f, x)-f(x)| \leq\left[1+\frac{1}{\varepsilon} \cdot \eta \cdot L(d(\cdot, x) ; x)\right] \cdot \omega(f, \varepsilon) .
$$

Thus, for $n \geq 1$ (which is the case for every coefficient of convex deformation) and for functions $f$ with the property mentioned above our theorem gives better constants in front of $\omega(f, \varepsilon)$. 
(ii) A statement akin to (i) holds with respect to Theorem 4 in Jiménez Pozo's paper [11].

As a further consequence of Theorem 3.1 we mention

THEOREM 3.11 (ef. Nishishiraho [20, Theorem 4]). Let $X$ be $a$ compact subset of a real pre-Hilbert space with inner product $(\cdot, *)$. Let $\varepsilon>0$.

If $L: C(X) \rightarrow C(X)$ is a positive linear operator, then for all $f \in C(X)$ and $x \in X$ there holds

$$
\begin{aligned}
|L(f, x)-f(x)| \leq|f(x)| & \cdot\left|L\left(1_{X}, x\right)-1\right| \\
& +\max \left\{|| L \|, \frac{1}{\varepsilon} \cdot L\left(d(\cdot, x)^{2} ; x\right)^{\frac{1}{2}} \cdot L\left(1_{X}, x\right)^{\frac{1}{2}}\right\} \cdot \tilde{\omega}(f, \varepsilon) .
\end{aligned}
$$

Here $d(x, y)=(x-y, x-y)^{\frac{1}{2}}$. If $L\left(1_{X}, x\right)=1$, then

$$
|L(f, x)-f(x)| \leq \max \left\{\|L\|, \frac{1}{\varepsilon} \cdot L\left(d(\cdot, x)^{2} ; x\right)^{\frac{1}{2}}\right\} \cdot \tilde{\omega}(f, \varepsilon) .
$$

Proof. Use Corollary 3.2 with $\Phi(x, y)=d(x, y)$ and $p=2$.

Under the assumptions of Theorem 3.11 it is also possible to give estimates similar to those in Theorem 3.4 or Corollary 3.5 .

\section{Applications}

As illustrations of our general results we consider three examples.

\subsection{A MODIFICATION OF LEHNHOFF'S TIMAN TYPE THEOREM}

Recently in a very interesting paper Lehnhoff [13] investigated the sequence of positive linear polynomial operators on $C[-1,1]$ given by

$$
H_{n}(f, x)=\frac{1}{\pi} \int_{-\pi}^{\pi} f(\cos (\arccos x+v)) \cdot K_{3 n-3}(v) d v
$$

with kernel

$$
K_{3 n-3}(v)=\frac{10}{n\left(11 n^{4}+5 n^{2}+4\right)}\left(\frac{\sin (n v / 2)}{\sin (v / 2)}\right)^{6}
$$

He showed that for all $f$ its image $H_{n} f$ is a polynomial of degree $3 n-3$ satisfying 


$$
H_{n}\left(\left(e_{1}-x\right)^{2}, x\right) \leq \frac{30}{11}\left(\frac{\sqrt{1-x^{2}}}{n}+\frac{|x|}{n^{2}}\right)^{2} \text {. }
$$

From Theorem 3.11 we see that for $f \in C[-1,1],|x| \leq 1$, and $\varepsilon>0$ one has

$$
\left|H_{n}(f, x)-f(x)\right| \leq \max \left\{1, \frac{1}{\varepsilon} \sqrt{\frac{30}{11}} \cdot\left\{\frac{\sqrt{1-x^{2}}}{n}+\frac{|x|}{n^{2}}\right)\right\} \cdot \tilde{\omega}(f, \varepsilon) .
$$

A proper choice of $\varepsilon$ then implies

$$
\left|H_{n}(f, x)-f(x)\right| \leq 1.66 \cdot \tilde{\omega}\left(f, \frac{\sqrt{1-x^{2}}}{n}+\frac{|x|}{n^{2}}\right) .
$$

However, the question for the best possible constant in a Timan type estimate in terms of $\tilde{\omega}(f, \cdot)$ still remains open.

\subsection{APPROXIMATION BY BIVARIATE BERNSTEIN-STANCU OPERATORS}

In his paper [23], Stancu investigated a generalization of the bivariate Bernstein operator, given by

$$
\left(L_{m, n}^{\langle\alpha, \beta\rangle} f\right)(x, y)=\sum_{i=0}^{m} \sum_{j=0}^{n} w_{m, i}^{\langle\alpha\rangle}(x) \cdot w_{n, j}^{\langle\beta\rangle}(y) \cdot f\left(\frac{i}{m}, \frac{j}{n}\right),
$$

where

$$
w_{p, k}^{\langle\gamma\rangle}(t)=\left(\begin{array}{l}
p \\
k
\end{array}\right)\left(\prod_{v=0}^{k-1}(t+v \gamma) \cdot \prod_{\nu=0}^{p-k-1}(1-t+v \gamma)\right) / \prod_{v=0}^{p-1}(1+v \gamma),
$$

$f \in C\left([0,1]^{2}\right),(x, y) \in X=[0,1]^{2}, n, m \geq 1, \alpha, \beta \geq 0$.

Obviously $L_{m, n}^{\langle\alpha, \beta\rangle}$ is a positive linear operator and hence Theorem 3.11 is applicable. Note that $L_{m, n}^{\langle\alpha, \beta\rangle}$ reprocudes ${ }^{1}{ }_{X}$. Using the euclidian metric $d$ on $X$ we have to consider

$$
\begin{aligned}
L_{m, n}^{\langle\alpha, \beta\rangle}\left(d(\cdot,(x, y))^{2} ;(x, y)\right) & \\
& =\sum_{i=0}^{m} \sum_{j=0}^{n} w_{m, i}^{\langle\alpha\rangle}(x) \cdot w_{n, j}^{\langle\beta\rangle}(y) \cdot\left[\left(x-\frac{i}{m}\right)^{2}+\left(y-\frac{j}{n}\right)^{2}\right] \\
& =\frac{1+\alpha m}{m(1+\alpha)} x(1-x)+\frac{1+\beta n}{n(1+\beta)} y(1-y) .
\end{aligned}
$$


Here some known facts about univariate Bernstein-Stancu operators were used (see, for example, [4]). Theorem 3.11 implies

$$
\left|\left(L_{m, n}^{\langle\alpha, \beta\rangle} f\right)(x, y)-f(x, y)\right| \leq \tilde{\omega}\left(f, \sqrt{\frac{1+\alpha m}{m(1+\alpha)} x(1-x)+\frac{1+\beta n}{n(1+\beta)} y(1-y)}\right) .
$$

For $\alpha=\beta=0$ this reduces to an inequality for bivariate Bernstein operators on $X$, namely

$$
\left|\left(B_{m, n} f\right)(x, y)-f(x, y)\right| \leq \tilde{\omega}\left(f, \sqrt{\frac{x(1-x)}{m}+\frac{y(1-y)}{n}}\right) .
$$

\subsection{SHEPHARD'S "METRIC INTERPOLATION"}

While the last two examples were mainly given in order to illustrate the magnitude of the constants in our general theorems, we now turn to an example that lives in metric spaces $(X, d)$.

Let $f$ be a real-valued function defined on $X$, and let $x_{1}, \ldots, x_{N}$ be a finite collection of distinct points in $X$. We assume further that for each $N$-tuple $\left(x_{1}, \ldots, x_{N}\right)$ we are given a finite sequence $\left(\mu_{1}, \ldots, \mu_{N}\right)$ of real numbers $\mu_{i}>0$. Then Shephard's method of "metric interpolation" is described by the operators $S_{N}$ given by

$$
\begin{aligned}
S_{N}(f, x) & =S_{x_{1}, \ldots, x_{N}}^{\mu_{1}, \ldots, \mu_{N}}(f, x) \\
& :=\left\{\begin{array}{r}
\sum_{i=1}^{N} f\left(x_{i}\right) \cdot\left(\prod_{j=1, j \neq i}^{N} d\left(x, x_{j}\right)^{\mu_{j}}\right) /\left(\sum_{i=1}^{N} \prod_{k=1, k \neq 2}^{N} d\left(x, x_{k}\right)^{\left.\mu_{k}\right)}\right) \\
\text { for } x k\left\{x_{1}, \ldots, x_{N}\right\}, \\
f\left(x_{i}\right) \text { otherwise. }
\end{array}\right.
\end{aligned}
$$

An alternative representation of the sum is

$$
\sum_{i=1}^{N} f\left(x_{i}\right) \cdot\left(\left(d\left(x, x_{i}\right)^{-\mu_{i}}\right) /\left(\sum_{i=1}^{N} d\left(x, x_{i}\right)^{-\mu_{i}}\right)\right) .
$$

It is immediately clear that $S_{N}$ is a positive linear operator on $C(X)$, say, and that $S_{N}\left(f, x_{i}\right)=f\left(x_{i}\right)$ for all $x_{i}$. Moreover, $S_{N}\left(1_{X}, x\right)=1$ for all $x \in X$. The $S_{N}$ 's were introduced by Shepard [21]; for further 
information see, for example, Gordon and Wixom [5].

In the sequel we restrict ourselves to the case where $1 \leq \mu=\mu_{1}=\ldots=\mu_{N}$. For the estimates given below we shall make use of the conventions $1 / \infty=0,1 / 0=\infty$, and $a+\infty=\infty$ for $a>0$.

THEOREM 4.1. If $\mu \geq 1$, then the Shepard operator $S_{N}^{\mu}$ satisfies for $f \in C(X)$ and $x \in X$ the inequalities

(i) $\left|S_{N}^{\mu}(f, x)-f(x)\right| \leq \tilde{\omega}\left(f, \tau_{N}(x)\right)$, where

$$
\tau_{N}(x):=\left(\sum_{i=1}^{N} d\left(x, x_{i}\right)^{-\mu+1}\right) / \sum_{i=1}^{N} d\left(x, x_{\eta}\right)^{-\mu},
$$

and

(ii) $\left|S_{N}^{\mu}(f, x)-f(x)\right| \leq \tilde{\omega}\left(f, X_{N}(x)^{1 / \mu}\right)$, where $\chi_{N}(x)$ is the harmonic mean of $d\left(x, x_{1}\right)^{\mu}, \ldots, d\left(x, x_{N}\right)^{\mu}$, that is

$$
x_{N}(x)=N \cdot\left(\sum_{i=1}^{N} 1 / d\left(x, x_{i}\right)^{\mu}\right)^{-1} .
$$

Proof. The proof of ( $i$ ) is an immediate consequence of the second representation of $S_{N}$ given above, of Theorem 3.1 and the fact that $S_{N}{ }^{X}=1_{X}$

Proof of (ii). Corollary 3.2 gives

$$
\left|S_{N}^{\mu}(f, x)-f(x)\right| \leq \max \left\{1, \frac{1}{\varepsilon} \cdot c\left(S_{N}^{\mu}, \Phi, I d, x\right)\right\} \cdot \tilde{\omega}(f, \varepsilon),
$$

where

$$
C\left(S_{N}^{\mu}, \Phi, \text { Id, } x\right)=\inf _{p \geq 1}\left\{S_{N}^{\mu}\left(\Phi(\cdot, x)^{p / q} ; x\right)^{1 / p}\right\} \text {, }
$$

and $\Phi$ is a function on $x^{2}$, such that for some $q \geq 1$ and $x, y \in X$ inequality $d(x, y)^{q} \leq \Phi(x, y)$ holàs. Choose $q=1$ and $\Phi(x, y)=d(x, y)$. Thus 


$$
C\left\{S_{N}^{\mu}, d, \operatorname{Id}, x\right\}=\inf _{p \geq 1}\left\{S_{N}^{\mu}\left(d(\cdot, x)^{p} ; x\right)^{1 / p}\right\} \leq\left[S_{N}^{\mu}\left(d(\cdot, x)^{\mu} ; x\right)\right]^{1 / \mu} .
$$

Using the second representation of $S_{N}^{\mu}$ it is immediately seen that

$$
S_{N}^{\mu}\left(d(\cdot, x)^{\mu} ; x\right)=\chi_{N}(x)
$$

Hence the above estimate reads

$$
\left|S_{N}^{\mu}(f, x)-f(x)\right| \leq \max \left\{1, \frac{1}{\varepsilon} \cdot x_{N}(x)^{1 / \mu}\right\} \cdot \tilde{\omega}(f, \varepsilon),
$$

and a proper choice of $\varepsilon$ for $x \neq x_{k}$ yields the above estimate. Due to the interpolation properties of $S_{N}^{\mu} f$ it remains true if $x$ is one of the interpolation points.

REMARK 4.2. (i) In the example to follow we shall apply the first assertion of Theorem 4.1. Although the second one is often useful for proving non-quantitative convergence theorems, the evaluation of ${ }^{\tau} N$ rather than of $x_{N}$ is of advantage for quantitative purposes. This observation corresponds to the well known fact that, when investigating the quantitative behavior of a sequence of positive linear operators $L_{n}$, the Cauchy-Schwarz inequality should only be used if $L_{n}(d(\cdot, x) ; x)$ and $L_{n}\left(d(\cdot, x)^{2} ; x\right)^{\frac{1}{2}}$ vanish of the same order.

(ii) Since the harmonic mean $x_{N}$ and the geometric mean $\Gamma_{N}$ of $N$ positive numbers are related by $\chi_{N} \leq \Gamma_{N}$, the second estimate in Theorem 4.1 implies

$$
\left|S_{N}^{\mu}(f, x)-f(x)\right| \leq \tilde{\omega}\left(f, \sqrt[N]{\prod_{l=1}^{N} d\left(x, x_{l}\right)}\right)
$$

Although the right hand side still shows that $S_{l}^{\mu}$ is an interpolation operator, it seems to be hardly of advantage to use geometric means (see also Remark 4.4).

In order to illustrate our general estimates for Shepard operators, we 
again consider a univariate case, namely $X=[0,1], d(x, y)=|x-y|$, $x_{i}=i / n$ for $0 \leq i \leq n$, and $1 \leq \mu \leq 2$. The cases $\mu>2$ were investigated by Somorjai [22] (see also Szabados [24] for $\mu=4$ ). For $x=[-1,1]$ and $x_{k}=\cos ((2 k-1) / 2 n) \pi, 1 \leq k \leq n$, see Hermann and Vértesi [6].

THEOREM 4.3. Let $X=[0,1], d(x, y)=|x-y|$, and $s_{n}^{\mu}$ be the Shepard operator based on the points $x_{i}=i / n, 0 \leq i \leq n$, and exponent $\mu$ with $1 \leq \mu \leq 2$. Then for all $f \in C[0,1]$ and all $n \geq 1$ we have

$$
\begin{aligned}
& \text { (i) }\left\|S_{n}^{1} f-f\right\|_{X} \leq \frac{n+1}{n} \cdot \tilde{\omega}(f, 1 / \ln (2 n+2)\}, \\
& \text { (ii) }\left\|S_{n}^{\mu} f-f\right\|_{X} \leq \frac{14}{2-\mu} \cdot \tilde{\omega}\left(f, 1 /(n+1)^{\mu-1}\right) \text { for } 1<\mu<2, \\
& \text { (iii) }\left\|S_{n}^{2} f-f\right\|_{X} \leq 19 \cdot \tilde{\omega}\left(f, \frac{\ln (n+1)}{n+1}\right) .
\end{aligned}
$$

Proof. (i) The second estimate in Theorem 4.1 shows that for all $x \in[0,1]$ we have

$$
\left|S_{n}^{l}(f, x)-f(x)\right| \leq \tilde{\omega}\left(f, X_{N}(x)\right)
$$

where $\chi_{N}(x)$ is the harmonic mean of the numbers $|x-(i / n)|, 0 \leq i \leq n$, that is,

$$
N \cdot\left(\sum_{i=0}^{n} 1 /|x-(i / n)|\right)^{-1} \text {, where } N=n+1
$$

Here we may assume that $x$ is none of the interpolation points since in this case the above estimate holds anyway. In order to determine how fast this expression tends to zero we have to find out how fast

$$
\frac{1}{N} \cdot \sum_{i=0}^{n} 1 /|x-(i / n)|
$$

tends to infinity: Let $i_{0}$ be defined by $i_{0} / n<x<\left(i_{0}+1\right) / n$. Then 


$$
\begin{aligned}
\frac{1}{N} \cdot \sum_{i=0}^{n} 1 /|x-(i / n)| & \geq \frac{n}{n+1} \cdot\left\{\sum_{i=0}^{i_{0}} 1 /\left(i_{0}+1-i\right)+\sum_{i=i_{0}+1}^{n} 1 /\left(i-i_{0}\right)\right\} \\
& \geq \frac{n}{n+1} \cdot\left\{\int_{1}^{i_{0}^{+2}} \frac{1}{x} d x+\int_{1}^{n-i_{0}+1} \frac{1}{x} d x\right\} \\
& =\frac{n}{n+1} \cdot \ln \left(\left(i_{0}+2\right) \cdot\left(n-i_{0}+1\right)\right\} \\
& \geq \frac{n}{n+1} \cdot \ln (2 n+2)
\end{aligned}
$$

which in turn shows that $x_{N}(x) \leq(n+1) / n \cdot 1 / \ln (2 n+2)$. This gives $(i)$.

(ii) and (iii). For the case $\mu>1$ we use the first inequality in Theorem 4.1 and thus have to estimate the quantity

$$
\sum_{i=0}^{n}\left|x-x_{i}\right| \cdot\left(\left(\left|x-x_{i}\right|^{-\mu}\right) /\left(\sum_{i=0}^{n}\left|x-x_{i}\right|^{-\mu}\right)\right) \text { for } x \leqslant\left\{x_{0}, \ldots, x_{n}\right\} .
$$

Let $z_{0}$ be defined by $\left|x-x_{\eta_{0}}\right|=\min \left\{\left|x-x_{2}\right|: 0 \leq z \leq n\right\}$. Then the last sum is less than or equal to

$$
\begin{aligned}
\left|x-x_{z_{0}}\right|^{\mu} \cdot \sum_{i=0}^{n}\left|x-x_{i}\right|^{1-\mu} & \leq \frac{1}{n}+\left(\frac{1}{n}\right)^{\mu} \cdot\left\{\sum_{i<\tau_{0}}\left|x-x_{i}\right|^{1-\mu}+\sum_{i>z_{0}}\left|x-x_{i}\right|^{1-\mu}\right\} \\
& \leq \frac{1}{n}+\frac{1}{n} \cdot\left\{\sum_{k=0}^{Z_{0}^{-1}}\left(\frac{1}{2}+k\right)^{1-\mu}+\sum_{k=0}^{n-z_{0}^{-1}}\left(\frac{1}{2}+k\right)^{1-\mu}\right\}
\end{aligned}
$$

where either of the sums may be empty. For $0 \leq \tau_{0} \leq n$ the quantity in accolades is less than or equal to

$$
2 \cdot{ }_{k=0}^{[n / 2]}\left(\frac{1}{2}+k\right)^{1-\mu} \leq 2 \cdot \begin{cases}2^{\mu-1}+\frac{1}{2-\mu} \cdot\left(\frac{n+1}{2}\right)^{2-\mu} \text { for } 1<\mu<2, \\ 2+\ln (n+1) \text { for } \mu=2 .\end{cases}
$$

This implies estimates (ii) and (iii) of Theorem 4.3.

REMARK 4.4. We follow up Remark 4.2 (ii). If in Theorem 4.3, with $\mu=1$ for example, we would have used geometric means instead of harmonic 
ones, the parameter in the modulus of continuity would have become $\left(\prod_{l=0}^{n}\left|x-\frac{l}{n}\right|\right)^{l / N}$, and, for an arbitrary choice of $N=n+1$ points $x_{0}, \ldots, x_{n}$ in the interval $[0,1],\left(\prod_{l=0}^{n}\left|x-x_{l}\right|\right)^{l / N}$ respectively. The quantity under the root is the modulus of a polynomial of degree $n+1$ with leading coefficient equal to one. As is well known, the sup norm of such a polynomial cannot become arbitrarily small. For instance, on $[-1,1]$ it is minimal for the Čebyšev polynomials $T_{n+1}$ where $\left\|\prod_{l=0}^{n}\left(x-x_{l}\right)\right\|_{[-1,1]} \geq 1 / 2^{n}$ for all possible choices of $x_{0}, \ldots, x_{n}$, even in $\mathbb{R}$. This means that $\left(\prod_{l=0}^{n}\left|x-x_{l}\right|\right)^{1 / N}$ cannot become arbitrarily small uniformly in $[0,1]$.

\section{References}

[1] H. Berens and G.G. Lorentz, "Theorems of Korovkin type for positive linear operators on Banach lattices", Approximation theory, 1-30 (Proc. Internat. Sympos. Austin, 1973. Academic Press, New York, San Francisco, London, 1973).

[2] В.К. Дзящז [V.K. Dzjadyk], Введение в теорию равномерного приближения фунжщий полинамами [Introduction to the theory of uniform approximation of functions by polynomials] (Izdat. "Nauka", Moscow, 1977).

[3] H. Gonska, "On Mamedov estimates for the approximation of finitely defined operators", Approximation theory III, 443-448 (Proc. Internat. Sympos. Austin, 1980. Academic Press, New York, San Francisco, London, 1980).

[4] Heinz H. Gonska and Jutta Meier, "Quantitative theorems on approximation by Bernstein-Stancu operators", Calcolo (to appear). 
[5] W.J. Gordon and J.A. Wixom, "Shepard's method of 'metric interpolation' to bivariate and multivariate interpolation", Math. Comp. 32 (1978), 253-264.

[6] T. Hermann and $P$. Vértesi, "On an interpolatory operator and its saturation", Acta Math. Acad. Sci. Hungar. 37 (1981), 1-9.

[7] M.A. Jiménez Pozo, "Sur les opérateurs linéaires positifs et la méthode des fonctions tests", C.R. Acad. Sci. Paris Sér. A 278 $(1974), 149-152$.

[8] M.A. Jiménez Pozo, "On the problem of the convergence of a sequence of linear operators", Moscow Univ. Math. Buzz. 33 (1978), no. 4, 1-8.

[9] M.A. Jiménez Pozo, "Deformation de la convexité et théorèmes du type Korovkin", C.R. Acad. Sci. Paris Sér. A 290 (1980), 213-215.

[10] M.A. Jiménez Pozo, "Convergence of sequences of linear functionals", 2. Angew. Math. Mech. 61 (1981), 495-500.

[11] Miguel A. Jiménez Pozo, "Quantitative theorems of Korovkin type in bounded function spaces", Constmuctive finction theory (Proc. Internat. Conf., Varna, Bulgaria, 1981. To appear).

[12] M.A. Jiménez Pozo y M. Baile Baldet, "Estimados del orden de convergencia de una sucesion de operadores lineales en espacios de funciones con peso", Cienc. Mat. (Havana) 2 (1981), 16-28.

[13] H.G. Lehnhoff, "A simple proof of A.F. Timan's theorem", J. Approx. Theory 38 (1983), $172-176$.

[14] Р.Г. Мамедов [R.6. Mamedov], "О порядке приблинения функций линейными полонительными операторами" [On the order of approximation of functions by linear positive operators], Dokl. Akad. Nauk SSSR 128 (1959), 674-676.

[15] K. Menger, "Untersuchungen über allgemeine Metrik", Math. Ann. 100 (1928), 75-163.

[16] B.S. Mitjagin and E.M. Semenov, "Lack of interpolation of linear operators in spaces of smooth functions", Math. USSR-Izv. 11 (1977), 1229-1266. 
[17] M.W. Müller and H. Walk, "Konvergenz- und Güteaussagen für die Approximation durch Folgen linearer positiver Operatoren", Constmuctive theory of functions, 221-233 (Proc. Internat. Conf. Varna, Bulgaria, 1970). Publishing House of the Bulgarian Academy of Sciences, Sofia, 1972).

[18] D.J. Newman and H.S. Shapiro, "Jackson's theorem in higher dimension", On approximation theory, 208-219 (Proc. Conf. Math. Res. Inst. Oberwolfach, 1963. Birkhäuser, Basel, 1964).

[19] Toshihiko Nishishiraho, "The rate of convergence of positive linear approximation processes", Approximation theory IV (Proc. Internat. Sympos. College Station, 1983. Academic Press, New York, San Francisco, London, to appear).

[20] Toshihiko Nishishiraho, "Convergence of positive linear approximation processes", Töhoku Math. J. (to appear).

[21] D. Shepard, "A two-dimensional interpolation function for irregularly spaced data", Proc. 1968 ACM National Conference, 517-524.

[22] G. Somorjai, "On a saturation problem", Acta Math. Acad. Sci. Hungar. 32 (1978), 377-381.

[23] D.D. Stancu, "Approximation of bivariate functions by means of some Bernstein-type operators", Multivariate approximation, 189-208 (Proc. Sympos. Durham, 1977. Academic Press, New York, San Francisco, London, 1978).

[24] J. Szabados, "On a problem of R. DeVore", Acta Math. Acad. Sci. Hungar. 27 (1976), 219-223.

[25] M. Wolff, "Über das Spektrum von Verbandshomomorphismen: in $C(X)$ ", Math. Ann. 182 (1969), 161-169.

University of Duisburg,

Department of Mathematics,

D-4100 Duisburg 1,

West Germany;
Department of Mathematical Sciences, Drexel University,

Philadelphia,

Pennsylvania 19104,

USA. 